\title{
Tunable magnetization dynamics in artificial spin ice via shape anisotropy modification
}

\author{
T. Dion $\odot,{ }^{1,2,{ }^{*}}$ D. M. Arroo, ${ }^{1}$ K. Yamanoi, ${ }^{3}$ T. Kimura, ${ }^{4}$ J. C. Gartside, ${ }^{2}$ L. F. Cohen, ${ }^{2}$ H. Kurebayashi, ${ }^{1,5}$ and W. R. Branford ${ }^{2}$ \\ ${ }^{1}$ London Centre for Nanotechnology, University College London, London WC1H OAH, United Kingdom \\ ${ }^{2}$ Condensed Matter Physics, Imperial College London, London SW7 2AZ, United Kingdom \\ ${ }^{3}$ Department of Physics, Science and Technology, Keio University, Yokohama 223-8522, Japan \\ ${ }^{4}$ Department Physics, Kyushu University, Fukuoka 819-0395, Japan \\ ${ }^{5}$ Department of Electronic and Electrical Engineering, University College London, London WC1E 7JE, United Kingdom
}

(Received 19 February 2019; revised manuscript received 2 August 2019; published 23 August 2019)

\begin{abstract}
Ferromagnetic resonance (FMR) is performed on kagome artificial spin ice (ASI) formed of disconnected $\mathrm{Ni}_{80} \mathrm{Fe}_{20}$ nanowires. Here we break the threefold angular symmetry of the kagome lattice by altering the coercive field of each sublattice via shape anisotropy modification. This allows for distinct high-frequency responses when a magnetic field is aligned along each sublattice and additionally enables simultaneous spin-wave resonances to be excited in all nanowire sublattices, unachievable in conventional kagome ASI. The different coercive field of each sublattice allows selective magnetic switching via global field, unlocking novel microstates inaccessible in homogeneous-nanowire ASI. The distinct spin-wave spectra of these states are detected experimentally via FMR and linked to underlying microstates using micromagnetic simulation.
\end{abstract}

DOI: 10.1103/PhysRevB.100.054433

\section{INTRODUCTION}

Artificial spin ice (ASI) initially garnered interest for its intriguing thermodynamic properties [1] arising from competing dipolar interactions at the vertices that may not be simultaneously satisfied [2-5]. This effect, termed geometric frustration, is contingent on identical interaction strengths between each moment at a vertex. As such, work in the field typically strives for identically fabricated magnetic nanowires to achieve parity between vertex interactions and allow observation of emergent phenomena such as massively degenerate ground states [6,7]. In recent years, focus on ASI has expanded to include fine microstate manipulation and highfrequency magnetization dynamics, with promising initial results of functionality for next generation computational technologies from information storage [8,9] to hardware based neural networks [10-12].

High-frequency dynamics in ASI [13-16] have been shown to be closely linked to the microstate, inviting functional designs exploiting ASI as a reconfigurable magnonic crystal (RMC) with microstate control used to tune the magnon response [17-20]. However, reliably accessing more than a handful of microstates beyond simple global field saturation has proved difficult, with either stochastic AC demagnetization protocols or complex rotating field sequences employed [21,22]. Total microstate control via individual nanowire reversal using magnetic force microscope tips has been demonstrated [23-25] but is time consuming and is unsuitable for device integration. Considering ASI as an RMC rather than a model system for geometric frustration, it becomes attractive to modify the aspect ratio and hence the shape anisotropy of each nanowire sublattice, forgoing

*troy.dion.16@ucl.ac.uk degenerate dipolar interactions for the microstate control afforded by the different coercive fields between each modified sublattice.

Recent studies have investigated individual weakly interacting vertices with asymmetric nanowire sizes $[26,27]$. They focus on the switching mechanisms in these systems using ferromagnetic resonance (FMR) and simulations but do not investigate the angular dependence. The studies are restricted to the primitive cell that forms an ASI only, so no conclusions about microstate dependence can be drawn.

The motivation of this work is to leverage shape anisotropy modification to achieve reliable microstate control facilitating reconfigurable access to multiple distinct spin-wave spectra within the ASI array. We break rotational symmetry by modifying the nanowire width of each sublattice to achieve this aim. The coercive field is strongly dependent on the aspect ratio of the nanowires providing simple but powerful control of the switching behavior as each sublattice switches at different applied global field. This allows a good compromise between the range of accessible states and simplicity of the switching methodology and is highly reliable. Additionally, we perform successive minor field loops, allowing access to an enhanced range of microstates without the need for complex protocols at the expense of reduced repeatability.

We use our microstate selection scheme to provide detailed investigation of magnetization dynamics in a broken threefold symmetry ASI including angular dependence measurements. Angular dependent FMR measurements in nanomagnets with threefold symmetry [13-15] show three FMR modes whose frequency depends on $|\cos (\theta)|$, where $\theta$ is the applied magnetic field angle, with each mode offset by $60^{\circ}$ when the applied field is small compared to the saturation field. For homogeneous nanowires only two of these modes can occur at the same field and frequency. Here we show that with shape anisotropy modification it is possible to bring all three modes 


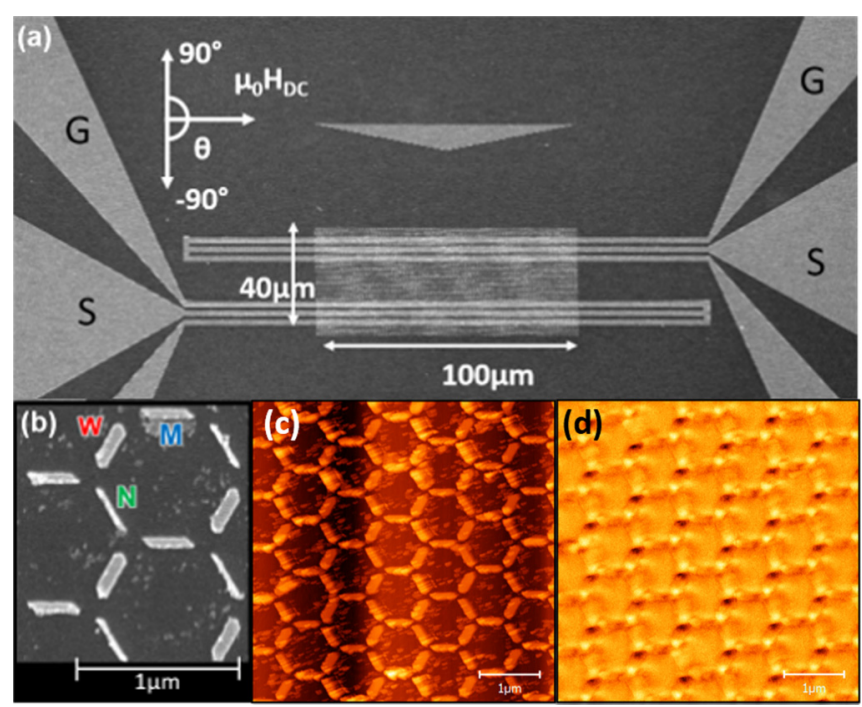

FIG. 1. SEM of device and 40 by $100 \mu \mathrm{m}$ ASI array. Two copper GSG type waveguides are deposited on top also via EBL with thickness of $200 \mathrm{~nm}$. The CPWs are connected to a VNA to measure the power reflection, $\mathrm{S}_{11}$. Angle $\theta$, used throughout script, are defined in the top left of the figure where $0^{\circ}$ is along the length of the medium nanowire. (b) SEM of the structure used to determine the width, lengths, and end-to-end distances of the nanowires. (c) AFM image for nanowire height characterisation. (d) MFM image showing nanowires obeying pseudo-ice-rule consistent with the presence of geometric frustration.

into resonance at the same applied field over a relatively small bandwidth.

The experiments performed measure the FMR of the sample using coplanar waveguides (CPWs) grown directly on top of the sample. A vector network analyzer (VNA) is used to measure the sample energy absorption. A scanning electron micrograph (SEM) of a finished device is shown in Fig. 1(a). Experimental data are compared with mumax3 micromagnetic simulations [28] which allow mode profile analysis and microstate visualization.

\section{SAMPLE GEOMETRY}

The ASI comprises three sublattices with different nanowire aspect ratios, fabricated $120^{\circ}$ to each other (see Fig. 1). Using electron beam lithography (EBL) and liftoff we have fabricated a permalloy kagome (or honeycomb) ASI where the nanowires in each orientation have lengths $l_{\text {nar }}=340 \pm 12 \mathrm{~nm}, l_{\text {med }}=320 \pm 12 \mathrm{~nm}$, and $l_{\text {wid }}=309 \pm$ $7 \mathrm{~nm}$, and widths $w_{\text {nar }}=54 \pm 10 \mathrm{~m}, w_{\text {med }}=86 \pm 8 \mathrm{~nm}$, and $w_{\text {wid }}=111 \pm 7 \mathrm{~nm}$; the mean nanowire height is $27.2 \pm$ $1.6 \mathrm{~nm}$ and the distance from nanowire end to center of vertex is $78 \pm 8 \mathrm{~nm}$. Throughout color versions of this paper the narrow, medium, and wide nanowires are colored green, blue, and red, respectively, and $0^{\circ}$ is defined along the axis of the medium nanowire. Figure 1(b) shows SEM of ASI on which the widths, lengths, and gap size were determined. Figure 1(c) shows atomic force micrograph (AFM) of the ASI structure from which the heights were determined. Figure 1(d) shows the phase contrast of a magnetic force micrograph
(MFM) exhibiting the pseudoice rule associated with kagome ASI where either two nanowires point in and one out of the vertex or vice versa. This demonstrates ice rules are obeyed by this system, despite unequal interaction strengths between various nanowires. Full descriptions of sample fabrication and characterization details are available in the Supplemental Material [29].

\section{RESULTS AND DISCUSSIONS}

\section{A. Field-frequency sweeps}

FMR is performed as a function of external magnetic field applied parallel to the length of each nanowire orientation. A broadband frequency reflection spectra $S_{11}$ is measured and the magnetic field is swept from positive to negative. Background is recorded by measuring $S_{11}$ in a large saturation field and this background is subtracted from subsequent measurements. All experiments are performed at room temperature. More detailed experimental procedures are described in the Supplemental Material [29].

Figures 2(a)-2(c) are the experimental broadband frequency power spectra as a function of applied magnetic field along the length of the (a) narrow, (b) medium, and (c) wide nanowires. The insets show the ASI structure orientation with respect to the applied field (white arrows). Figures 2(d)-2(f) are the corresponding micromagnetic simulation results and the insets show the geometry used where periodic boundary conditions have been applied. A magnetic field is applied to the simulated structure and it is allowed to relax to its ground state for all magnetic field values and orientations to compare with experiments. These ground state files are then used as inputs to simulate the FMR experiment. A broadband frequency oscillating field is applied via a sinc pulse with cutoff frequency $20 \mathrm{GHz}$ and amplitude of $0.1 \mathrm{Oe}$. The magnetization is recorded every 25 ps for a total time of $10 \mathrm{~ns}$ and the ground state magnetization is subtracted from all subsequent time steps to extract the dynamics. A fast Fourier transformation (FFT) is then applied to every cell in the mesh to convert to the frequency domain. Spin-wave eigenmodes are calculated by integrating over all micromagnetic cells. Mode profiles are generated by plotting the integrated Fourier amplitude for a given frequency range for each cell as a heat map. This provides spatial information about the modes and we refer to these plots as power pixel plots throughout the paper (further simulation details can be found in the Supplemental Material [29]). A more complete treatment of how micromagnetic simulations generate FMR spectra and mode profiles may be found in Ref. [30]. Figures 2(g)-2(i) show the spatial power pixel plots at resonant frequencies for $1.5 \mathrm{kOe}$ field aligned along the length of each nanowire. $1.5 \mathrm{kOe}$ is chosen as the modes are well separated and easier to identify than regions where modes coexist at similar frequencies. The modes show strong spatial localization which is well understood for nonellipsoidal nanomagnets [31]. These plots are used to assign the mode profiles exhibiting bulk mode behavior in Figs. 2(a)-2(f). No avoided crossing is observed between bulk modes which suggests there is no dipole-dipole coupling between different nanowires at high frequencies. There are some modes below $7 \mathrm{GHz}$ that do not appear in 


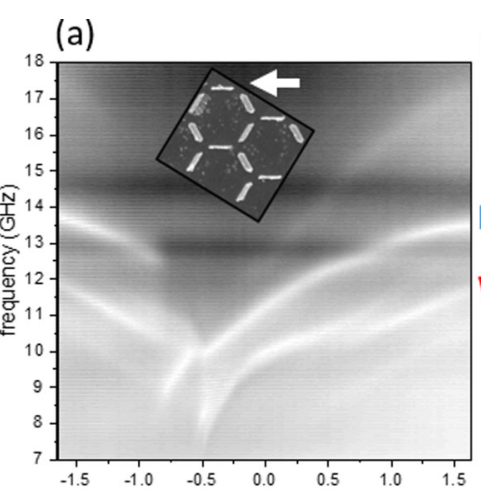

(d)

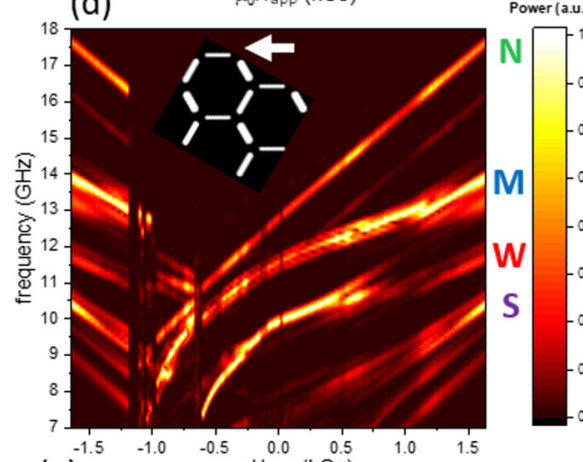

(g)
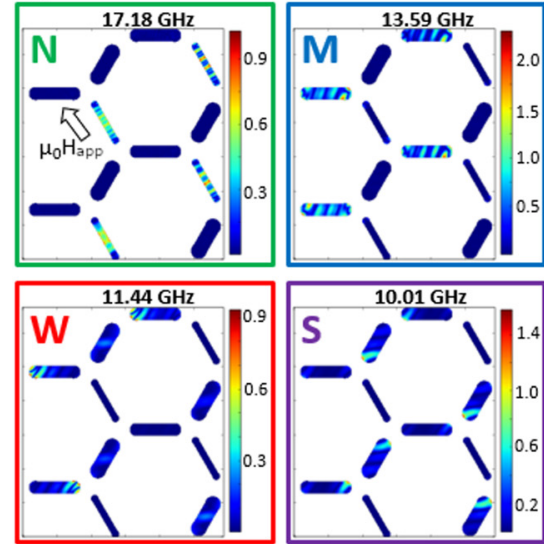

(j)
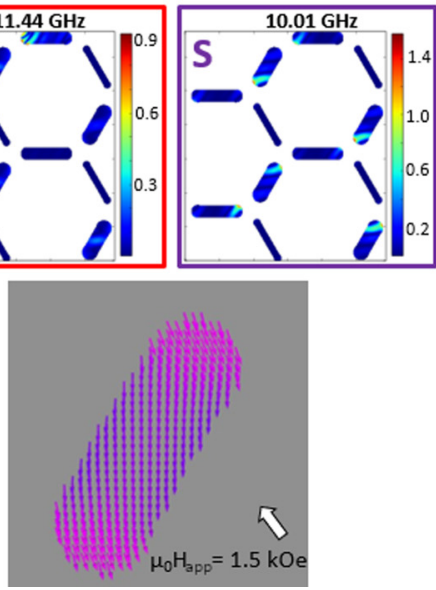

(b)

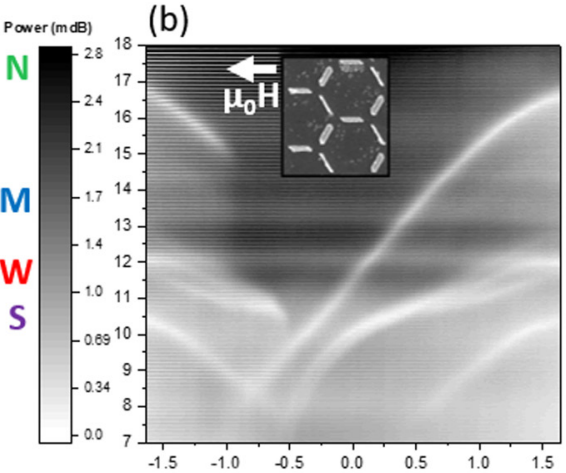

(e)

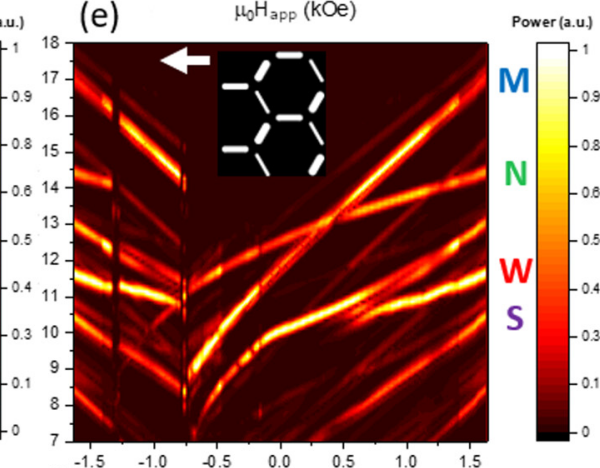

(h)

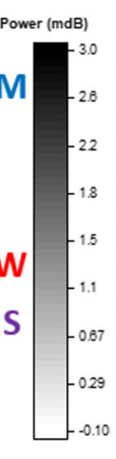

(c)

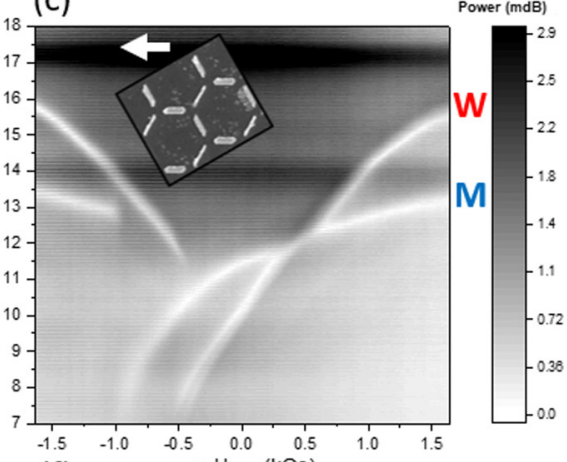

(f)

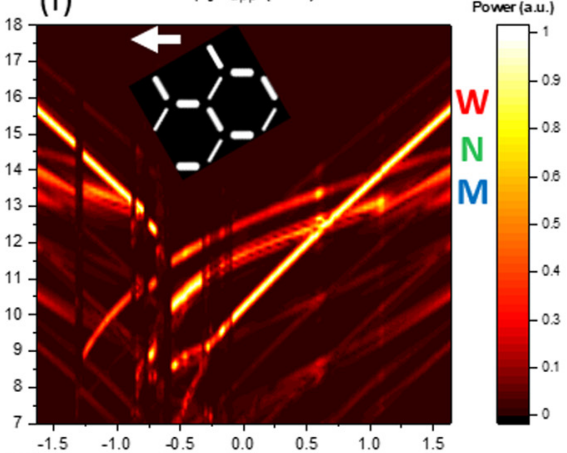

(i)
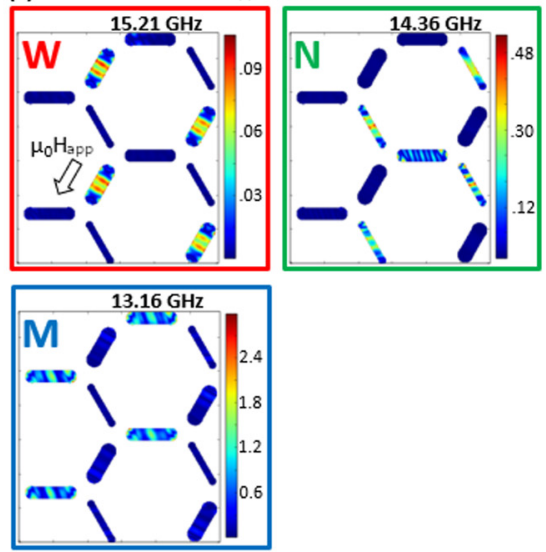

(I)

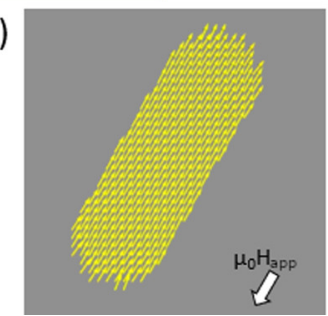

FIG. 2. (a), (b), and (c) are experimental magnetic field sweeps from positive to negative aligned along the length of the narrow, medium, and wide nanowires, respectively, as illustrated by the inset and arrow. (d), (e), and (f) are the corresponding micromagnetic simulations with inset showing geometry used with periodic boundary conditions applied. The labeled modes in Figs. (a)-(f) are illustrated in the spatial pixel power plots in (g), (h), and (i) at $1.5 \mathrm{kOe}$ along the direction of the white arrow. The N, M, and $\mathrm{W}$ modes are located in the bulk of the narrow, medium, and wide nanowires, respectively. The S mode is the result of magnetization curling at the ends of the wide nanowire. (j) and (k) are the magnetization profiles of the wide nanowire at $1.5 \mathrm{kOe}$ offset by $\pm 60^{\circ}$ resulting in the $\mathrm{S}$ mode. (1) is the magnetization profile of the wide nanowire when the field is aligned along the length in which case the $\mathrm{S}$ mode is not present. The N mode in (h) and (i) is not detected experimentally due to relatively lower volume compared to the other two nanowires. This fact combined with the S mode only occurring in (g) and (h) explains the different mode numbers for each of the three cases. 
the experimental data which are localized at nanowire ends, so-called edge modes, with significantly lower power and are not typically seen in experiments [26,27] so are not shown in simulations for clarity. We note that there does appear to be some mode hybridization within the wide nanowire in Figs. 2(d) and 2(e) indicated by avoided crossing around $0.6 \mathrm{kOe}$, however, a full investigation is outside the scope of the current work.

The frequency of precession is dependent on the total effective field, $H_{\text {tot }}=H_{\text {app }}|\cos (\theta)|+H_{\text {sha }}$, where $H_{\text {app }}$ is the applied magnetic field at in-plane angle $\theta$ [defined along the length of the medium nanowire, see Fig. 1(a)] and $H_{\text {sha }}$ is the shape anisotropy field which is a consequence of the demagnetizing field [32] and is fixed along the axis of each nanowire. The maxima occur when the field is aligned along the length of the nanowire and the minima when the field is perpendicular due to the strong dependence of $H_{\text {tot }}$ on the shape anisotropy at these length scales. Figure 2(a) is for the field aligned along the length of the narrow nanowire. The highest frequency mode is attributed to this nanowire which at $1.5 \mathrm{kOe}$ has a frequency of $17.35 \pm 0.25 \mathrm{GHz}$ compared to $17.24 \pm$ $0.10 \mathrm{GHz}$ simulated and is labeled $\mathrm{N}$. The wide and medium nanowires are both $60^{\circ}$ relative to the applied field, therefore, their mode profiles, $\mathrm{W}$ and $\mathrm{M}$, respectively, have roughly the same gradient. The anisotropy field is higher for the medium nanowire which causes an offset in frequency compared to the wide one. An additional mode at lower frequency is observed experimentally, accounted for by consideration of the wide nanowire magnetization profile shown in Fig. 2(j). The wide nanowire exhibits significant magnetization curling towards the ends of the nanowire forming an S-like profile. This $\mathrm{S}$ mode should not be confused with the edge modes previously discussed which occur at lower frequencies. They are more localized to the very edge of the nanowire than is observed for the $\mathrm{S}$ mode. The power pixel plot in Fig. 2(g) labeled S shows the resonance is located towards the nanowire ends in the curled magnetization region but not at the edge. Theoretical calculations for ASI often assume Ising like behavior so this work experimentally corroborates previous simulation studies on bent magnetizations [17]. For wide nanowires the Ising spin assumption is not valid and micromagnetic simulations are required to capture the physics completely. The $\mathrm{N}$ mode has relatively lower power absorption due to a smaller volume of the narrow nanowire compared to the other two nanowires. Discontinuities in the mode profiles in Fig. 2(d) between -0.5 and $1.3 \mathrm{kOe}$ are due to the switching of sublattices. These are not observed in Fig. 2(a) because the simulated geometry has homogeneous nanowire dimensions within a given sublattice and therefore the same coercive field. This means all the nanowires within the sublattice switch simultaneously. In the real sample there will be a distribution of coercive field due to imperfections caused by the fabrication process. A more gradual switching of nanowires smears out the abrupt changes predicted by simulations. These results are consistent with the onset of mode softening which occurs near the switching field of each nanowire $[26,33]$ where the frequency tends to zero. By comparing where the frequencies of each mode in Figs. 2(a)-2(c) tend to zero the switching fields can be estimated. Due to the faint contrast for the narrow nanowire an extraction method is required to extract the coercive field. The frequency response should be symmetric at high positive and negative fields. A reflection transformation along the central $y$ axis followed by a subsequent subtraction of the transformed data from the initial data increases the contrast allowing the switching field to be determined more accurately. A visualization of this process is available in the Supplemental Material [29]. The coercive fields of each sublattice are $H_{\mathrm{c}}^{\text {nar }}=1300 \pm 20 \mathrm{Oe}, H_{\mathrm{c}}^{\text {med }}=960 \pm 10$, and $H_{\mathrm{c}}^{\text {wid }}=450 \pm 10$. The large difference in coercive field of each sublattice is what allows reliable selective switching behavior.

Figure 2(b) is field aligned along the medium nanowire axis and as expected the highest frequency mode is located on medium nanowires with a frequency of $16.45 \pm 0.25 \mathrm{GHz}$ compared to $16.58 \pm 0.10 \mathrm{GHz}$ simulated. This is lower than the $\mathrm{N}$ mode in Fig. 2(a) at the same field and frequency which is consistent with the lower shape anisotropy. The W mode is roughly the same in Figs. 2(a) and 2(b) because in each case the applied field is $60^{\circ}$ offset so the effective field is the same. The $\mathrm{S}$ mode which is the result of curled magnetization at the ends of the wide nanowire is observed with a similar frequency as in Fig. 2(a). However, the direction of the curling is reversed which can be seen in the power pixel plot in Fig. 2(h) and the magnetization profile in Fig. 2(k).

Figure 2(c) shows the experimental results with field aligned along the length of the wide nanowire. The frequency of the $\mathrm{W}$ mode at $1.5 \mathrm{kOe}$ is consistently lower than the $\mathrm{N}$ and $\mathrm{M}$ modes for the other two alignments with a value of $15.40 \pm 0.10 \mathrm{GHz}$ compared to $15.38 \pm 0.10 \mathrm{GHz}$ simulated. The $\mathrm{M}$ mode is roughly the same as it is in Fig. 2(a) as expected. The $\mathrm{S}$ mode is not present for this case because as Fig. 2(i) shows, the magnetization of the wide nanowire exhibits no significant divergence at the ends.

Interestingly the number of modes detected experimentally in each case is different. This is the result of the interplay of several factors. In Fig. 2(a) a mode from the bulk of each sublattice plus the $\mathrm{S}$ mode from the wide nanowire results in four modes. The absence of the $\mathrm{N}$ mode in Figs. 2(b) and 2(c) is due to lower volume and therefore reduces the number of modes in these cases. Therefore, for Fig. 2(b) there are only three modes. Note that the mode is not deactivated but merely suppressed below the noise floor of the experimental apparatus. Finally, because the $\mathrm{S}$ mode requires the field to be misaligned with the wide nanowire, the number of modes in the case Fig. 2(c) is reduced to two. This effect on the other hand is a real deactivation of the $\mathrm{S}$ mode. This mode number flexibility is a direct result of controlling the size of the individual sublattice element sizes. Reducing the size means that when the field is not aligned with that nanowire the resonance can be suppressed. Increasing the size can create modes that are only present when the field is misaligned with the length of that sublattice providing a mode that can be activated or deactivated. This demonstration may be of use in the design of future spintronics or magnonics applications that require control over mode number particularly in microwave filtering applications where the forbidden and allowed frequency ranges can be manipulated. This work suggests a potential way of changing the number of detected modes simply by changing the angle of the applied external field. In a conventional ASI the exact same spin-wave spectra would 

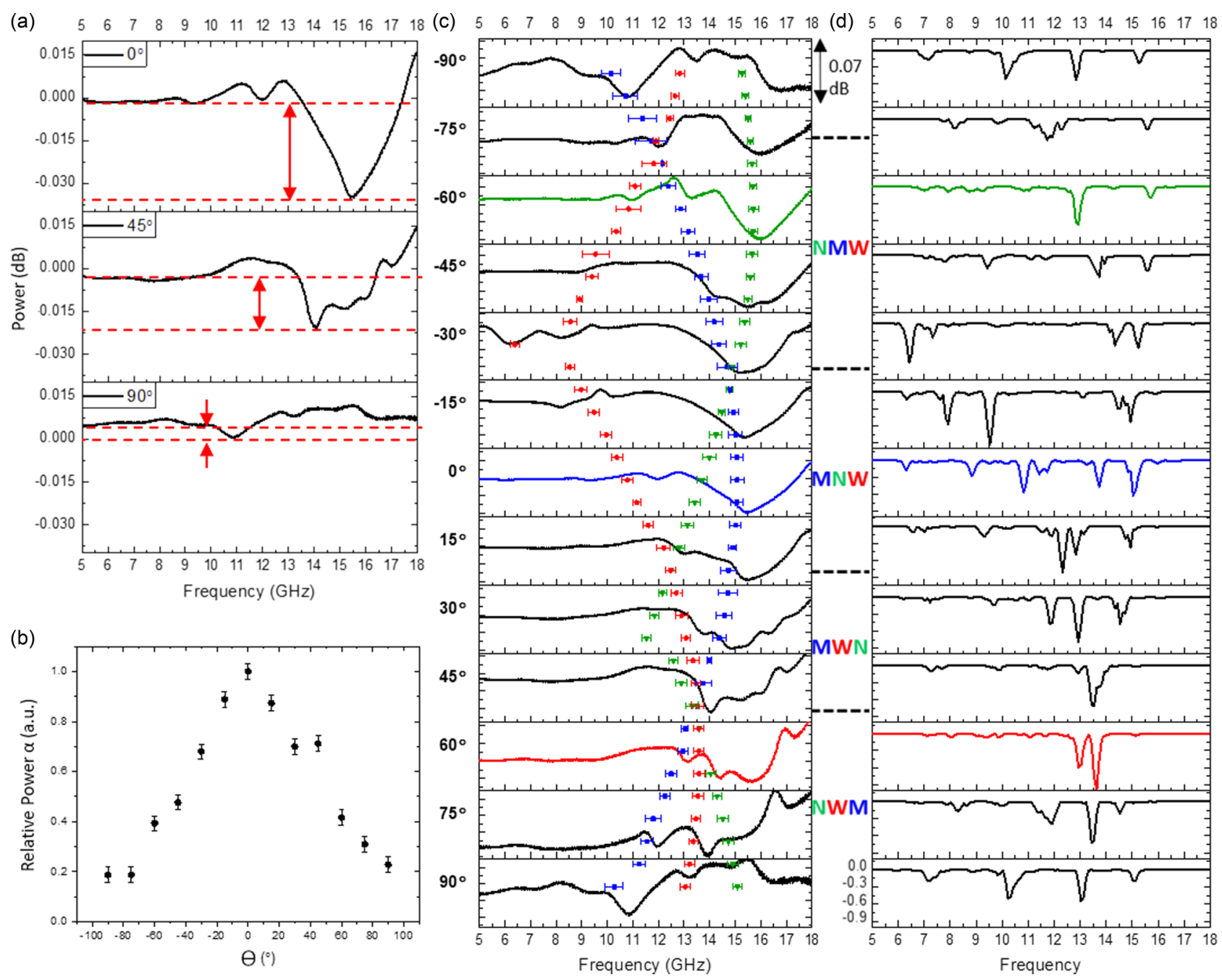

FIG. 3. (a) is the experimental frequency dependence of the absorbed power for three angles. The M-mode peak height is measured as shown by the dotted lines and arrows. These are normalized by the peak height at $0^{\circ}$. (b) shows the normalized power, $\alpha$, for all angles which varies sinusoidally. The errors are estimated from the variation in the power of the broadband frequency and error propagation is calculated. There is a relative increase in the peak height at $45^{\circ}$ which occurs where the modes are brought closer together as can be seen in (c) which shows the full angular dependence where each result is divided by $\alpha$ so they are displayed more clearly. The green triangles, blue squares, and red circles show the peak positions and error bars show the full width at half maximum (FWHM) for the narrow, medium, and wide modes, respectively. Colored spectra indicate when the field is aligned along a particular nanowire. (d) is the simulated angular dependence which is normalized to the maximum peak across all spectra so the relative peak size as a function of the angle is discernible. Each of the four possible permutations of nanowire frequency ordering regions are indicated between (c) and (d) and the dotted lines indicate the mode positions switching. The NMW region is where the four mode case is possible in other regions the $\mathrm{N}$ mode is too weak to be detected. There is a switching of the $\mathrm{M}$ and $\mathrm{N}$ modes at $25^{\circ}$ where the MNW region begins where it is possible to have three modes. Next is the MWN region which contains the coincidental angle where the three modes come close together. Finally the NWM mode where it is possible to have a case with two detectable modes. The $\mathrm{S}$ mode is suppressed here as the field is more closely aligned with the length of the wide nanowire

be observed regardless of which sublattice the field is aligned with.

The highest frequency modes at $1.5 \mathrm{kOe}$ are $17.24 \pm$ $0.10 \mathrm{GHz}, 16.45 \pm 0.25 \mathrm{GHz}$, and $15.40 \pm 0.10 \mathrm{GHz}$ when the field is aligned along the length of the narrow, medium, and wide nanowires, respectively, corresponding to a broad range of $1.84 \mathrm{GHz}$. This provides scope for manipulating magnetization dynamics by modifying the elements that form the structures to gain richer spin-wave spectra as well as the overall geometry.

\section{B. Angular dependence}

An angular dependence measurement is useful as it can clearly illustrate the effect of the shape anisotropy on the magnetization dynamics. The angular dependence of the sample is measured at $1 \mathrm{kOe}$ after being saturated and background spectrum recorded.

Figure 3(a) shows the power spectra taken at three different angles. This demonstrates how the coupling strength between the magnetization and oscillating field generated by CPW drops significantly as they are no longer in the optimum 
perpendicular orientation. To characterize the coupling the M-mode peak height is measured as indicated by the dotted line and arrow. A baseline is subtracted using the relatively flat part of the spectra below $9 \mathrm{GHz}$ and the maximum power absorption is measured at the M-mode peak. Each peak is then normalized by the largest peak at $0^{\circ}$ defining relative peak height $\alpha$. The relative peak height as a function of angle is plotted in Fig. 3(b) which varies sinusoidally. There is an increase in the relative peak height at $45^{\circ}$ which happens to be where the modes are brought closer together which is discussed further later. Figure 3(b) is used to normalize the experimental data by dividing each spectra by $\alpha$ so the peaks in Fig. 3(c) can be seen more clearly, particularly for angles far from $0^{\circ}$. The difference between the maximum and minimum value on each scale is $0.07 \mathrm{~dB}$. The peak positions obtained from simulations [Fig. 3(d)] are overlaid on the experimental data where the $\mathrm{N}, \mathrm{M}$, and $\mathrm{W}$ modes are represented by green triangles, blue squares, and red circles, respectively. The error bars denote the full width at half maxima (FWHM). The colored traces indicate when the field is aligned along the length of a particular nanowire.

The narrow nanowire has the highest frequency response followed by the medium and wide nanowires, which is expected since $H_{\text {sha }}^{\text {nar }}>H_{\text {sha }}^{\text {med }}>H_{\text {sha }}^{\text {wid }}$. Conversely, the wide nanowire shows the largest change in frequency with angle and the narrow one the lowest. Both observations are consistent with differences in shape anisotropy and previous work shows that aspect ratios of ellipsoids influence the magnetization dynamics with frequency highest when the field is aligned with the easy axis and lowest when aligned with the short (hard) axis [34]. We define the angle between the average magnetization vector and the long (easy) axis of the nanowire as $\Phi$. When the field is applied along the easy axis of a nanowire $\Phi$ is less than $0.5^{\circ}$ for all three nanowire geometries which are extracted from micromagnetic simulations at $1 \mathrm{kOe}$. However, when the applied field is along the hard axis there is a considerable difference in $\Phi$ for each different nanowire. For the wide nanowire $\Phi_{\text {wid }}=39.12^{\circ}$ and for the narrow $\Phi_{\text {nar }}=22.73^{\circ}$. Taking the ratio of these two angles gives a value of 1.72 . We now define $\Delta$ as the difference between the maximum and minimum frequency of each nanowire in the angular dependence which is along the easy and hard axis, respectively. $\Delta_{\text {nar }}=3.38 \mathrm{GHz}$ and $\Delta_{\text {wid }}=6.27 \mathrm{GHz}$. Taking the ratio of these two values gives 1.64 which is close to the ratio found of the two $\Phi$ values. The discrepancy is likely due to the fact that the modes are centrally localized but the average magnetization vector is calculated across the entire nanowire so end curling may cause an overestimation of $\Phi$. The surrounding nanowires may also contribute to small changes to $\Phi$. The stronger shape anisotropy in the narrow nanowire prevents the magnetic moments from rotating to align with the applied field. This explains why the narrow nanowire exhibits less variation in frequency as a function of the applied field angle compared to the wide nanowire because the total effective field changes less compared to the wide nanowire. The shape anisotropy is weaker for the wide nanowire and the magnetic moments can rotate more in the same applied field and thus there is larger variance in the frequency as a function of angle. Details on how $\Phi$ is determined from micromagnetic simulations can be found in the Supplemental Material [29].

There exist four regions in the angular dependence that are characterized by the order in which the modes occur in frequency. Mathematically there are a possible six permutations of the N, M, and $\mathrm{W}$ modes. Two of these permutations, namely, the ones which have the $\mathrm{W}$ mode as the highest frequency (WMN and WNM) do not occur. Between $51^{\circ}$ and $-75^{\circ}$ we have the permutation NWM (from highest to lowest in frequency). This region is the widest spanning $54^{\circ}$. There is a crossing point at $-75^{\circ}$ where the $\mathrm{W}$ and $\mathrm{M}$ modes switch places forming the region NMW which persists up to $-25^{\circ}$ with a width of $50^{\circ}$. In the next region the $\mathrm{N}$ and $\mathrm{M}$ modes swap places forming the MNW region and this persists to $20^{\circ}$ with a width of $45^{\circ}$. In the final region the $\mathrm{W}$ and $\mathrm{N}$ modes swap forming the MWN region up to $51^{\circ}$ which is the narrowest with width $31^{\circ}$. At the $51^{\circ}$ angle the three modes coincide and the $\mathrm{N}$ and $\mathrm{M}$ modes swap to return to the first region discussed. Each experiment from the previous section falls within a different region as described above. Region NWM contains the case where four modes are possible. This is because this is close to alignment with the narrow sublattice so the $\mathrm{N}$ mode is detectable in this region. Because this orientation is far away from alignment with the wide nanowire the $\mathrm{S}$ mode is observable. The NMW region is far away from alignment with the narrow nanowire and therefore the power of the $\mathrm{N}$ mode is suppressed and not detected. Since there is closer alignment with the wide nanowire the $\mathrm{S}$ mode is not activated so the number of modes is reduced from four to two in this region. In the $\mathrm{MNW}$ region the $\mathrm{S}$ mode is present but the $\mathrm{N}$ mode is not, meaning that there are three modes. The final region, MWN, is where the modes can be brought close together where only one mode is detected. Hence, the number of modes can be changed through one to four by changing the applied field angle.

The experimental data show much broader peaks relative to simulation. This is due to the micromagnetic damping parameter being set to zero in simulation to aid peak-position identification. A real sample experiences intrinsic material damping in addition to extrinsic damping caused by fabrication imperfections and thermal effects. Modelling temperature as a stochastic field at each time step causes slight fluctuations in resonant frequency for each nanowire, resulting in linewidth broadening but no change in the resonant peak position, $f$, for the entire ASI array $[13,15]$. ASI comprising nanowires of these dimensions can be safely approximated as an athermal system [35,36] far below the blocking temperature and as such no spontaneous magnetization reversals are expected. It should also be noted that the background elimination procedure has failed to completely rid the spectra of artifacts. Interpretation of features above $16 \mathrm{GHz}$ must be taken with care, however, simulations do not predict any mode structure beyond this frequency.

At $45^{\circ}$ the resonant frequencies of all nanowires are brought close together and the experimental data in Fig. 3(b) shows an anomalously larger relative peak height. If all nanowires were equally sized it would not be possible to resonate all nanowires at the same frequency because three $|\cos (\theta)|$ functions offset by $60^{\circ}$ cannot coincide 
(a)

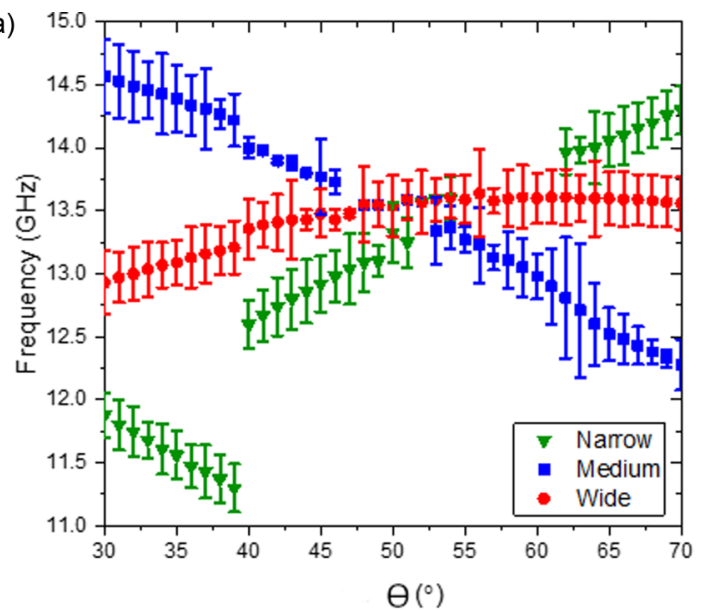

(c)

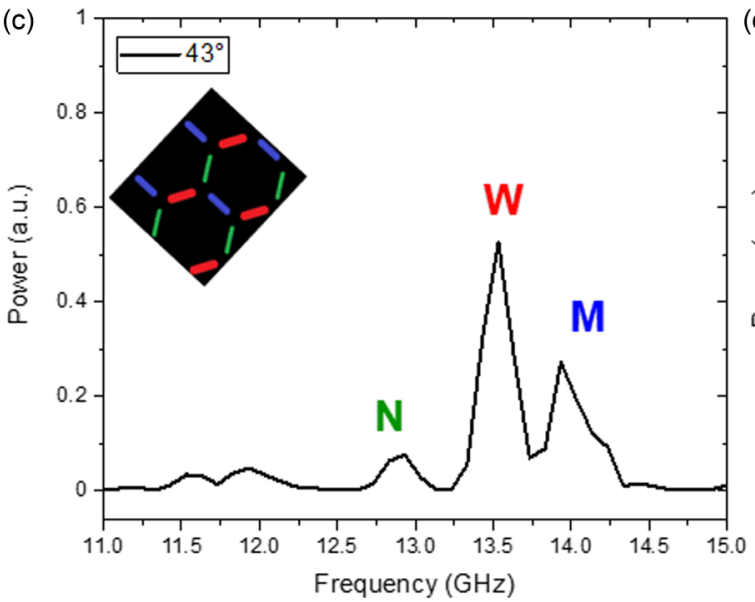

(e)

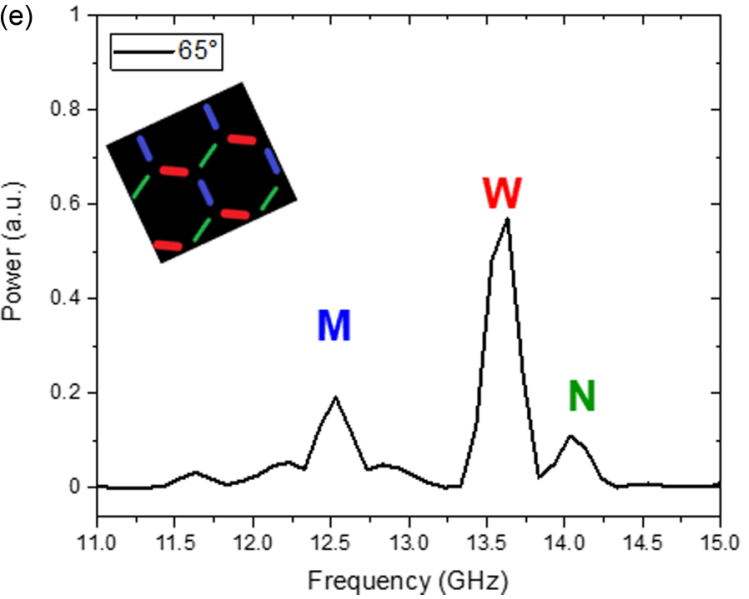

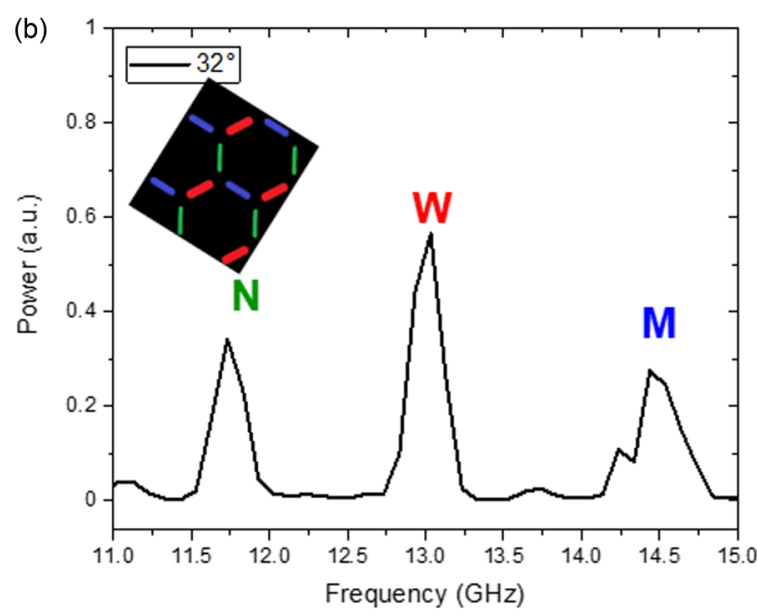

(d)

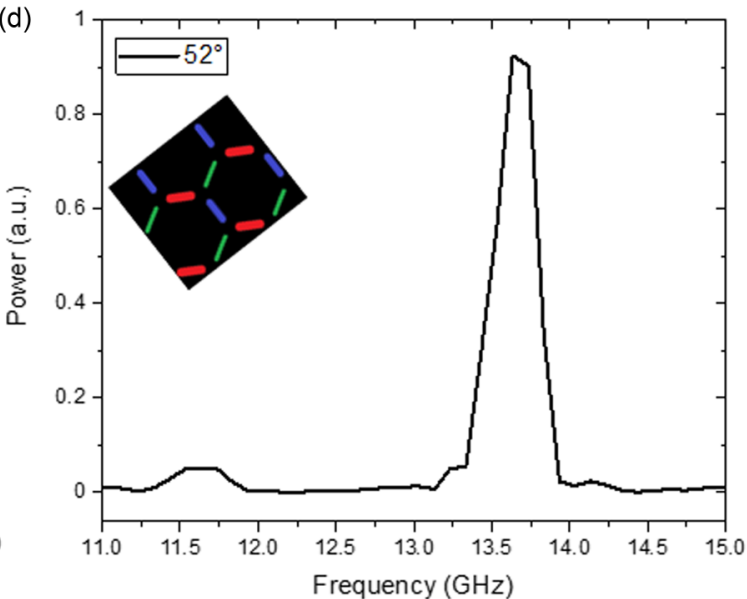

(f)

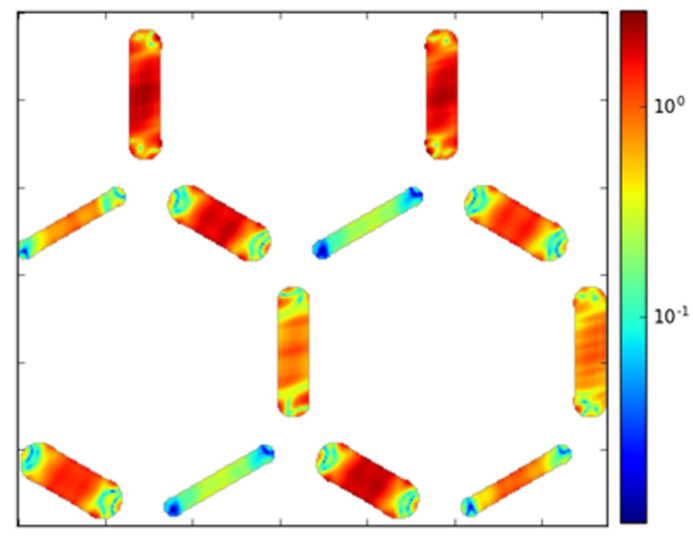

$13.35-13.95 \mathrm{GHz}$

FIG. 4. (a) shows results from angular dependent simulation around the region where the three modes $\mathrm{N}$ (green triangles), M (blue squares), and W (red circles) are brought close together. The error bars show the FWHM. (b)-(e) illustrate the modes coming together and then apart again as the angle is varied. The insets show the relative orientation of the ASI. (d) shows the modes coming close together at $52^{\circ}$ with an increase in relative amplitude. The full bandwidth (defined as between where the resonance starts and ends) of the cumulative peak is used to integrate the power pixel map in (f) which shows resonance in all sublattices over a $600 \mathrm{MHz}$ range.

simultaneously at any angle. To investigate this further angular dependent simulations were performed for every degree between $30^{\circ}$ and $70^{\circ}$ and the results are shown in Fig. 4(a). There is significant overlap of the peaks at $52^{\circ}$. Figures 4(b)-4(e) show the evolution of the peaks as they move closer together then apart again (a short video of the mode evolution as the angle is swept is available on- line [29]). It is also apparent that the relative peak intensity increases at $52^{\circ}$ which corroborates the experimental findings in Fig. 3(b) which shows the relative peak height increase at $45^{\circ}$. Figure 4(f) shows the power pixel plot for the peak in Fig. 4(d) demonstrating simultaneous resonance in all three nanowires over a $600 \mathrm{MHz}$ bandwidth (video of simulation driven at the resonance frequency is available online [29]. This 
(a)

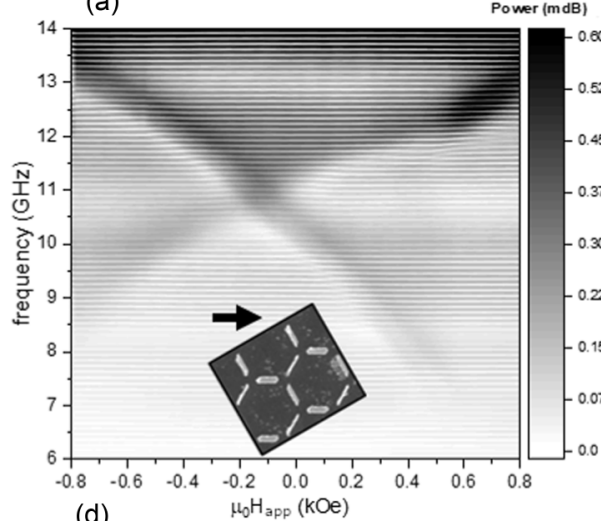

(d)

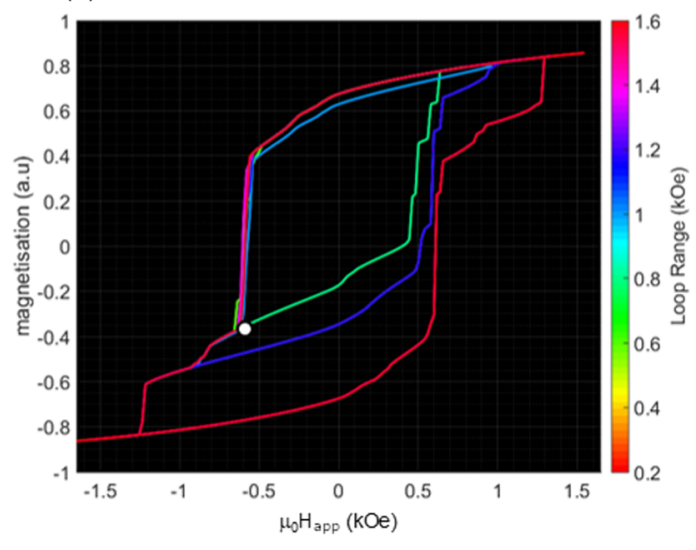

(g)

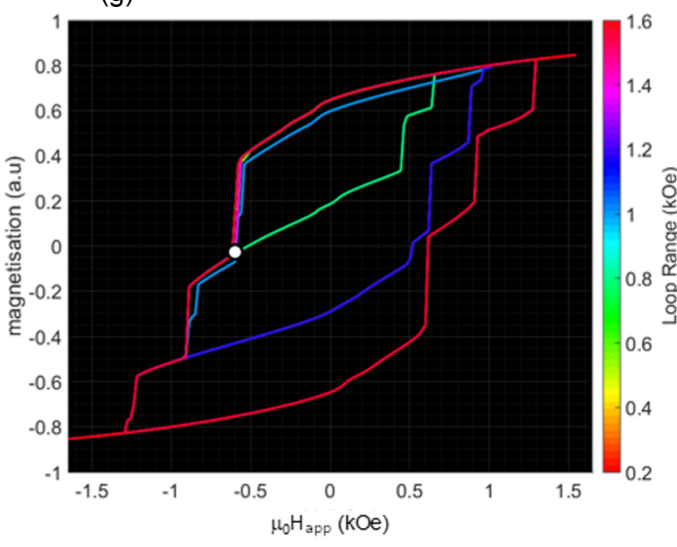

(b)

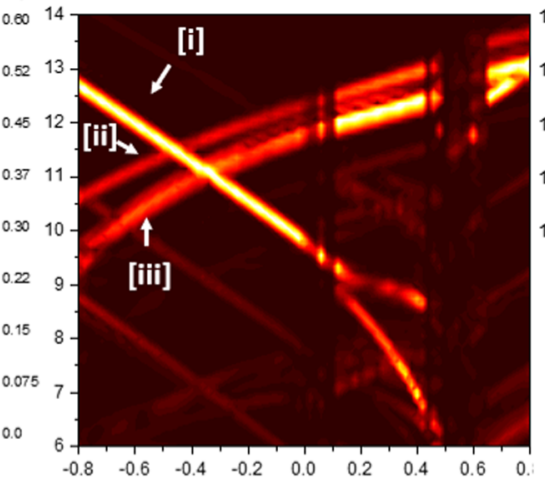

(e)

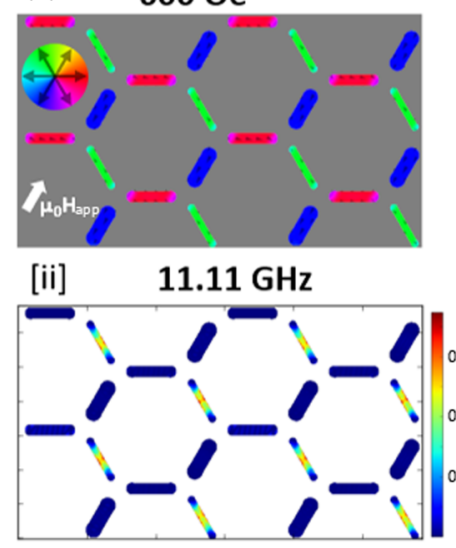

(h)

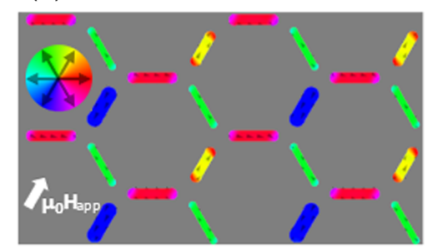

[V]

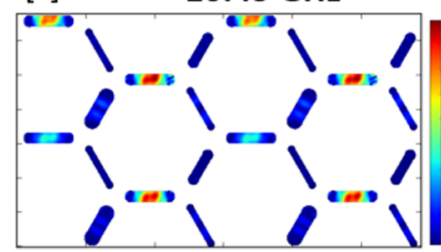

(c)

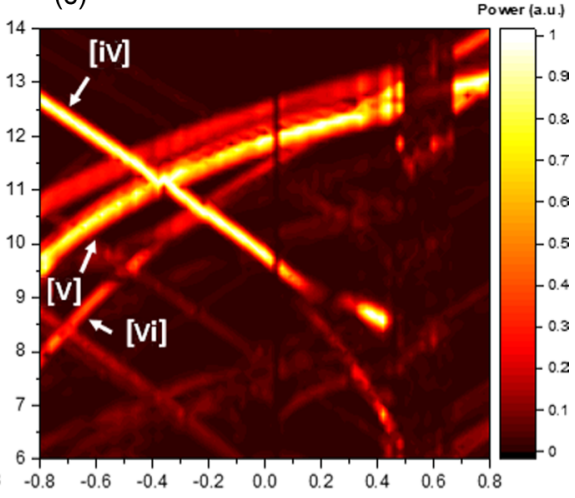

(f) [i]

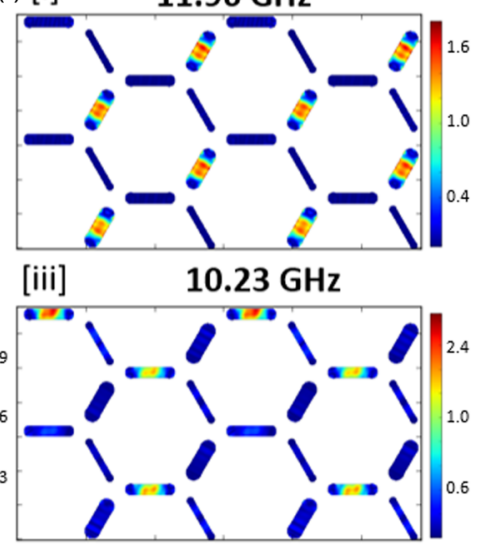

(i) [iv] $\quad 11.96 \mathrm{GHz}$
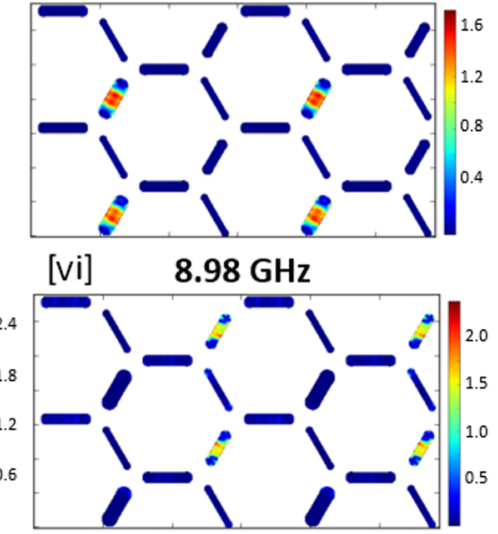

FIG. 5. A minor loop field protocol is performed starting from -200 to 200 Oe and increasing by 200 Oe after each minor loop up to $1 \mathrm{kOe}$ along the length of the wide nanowire. (a) is the experimental result of the fourth minor loop with range -800 to 800 Oe. The field is swept from negative to positive as illustrated in the inset. (b) is the simulated FMR for the current ASI structure. The labeled modes correspond to the power pixel plots in (e). (c) is the simulation result where the width of half of the wide nanowires are reduced by $35 \mathrm{~nm}$ which introduces a new mode [vi]. (d) and (g) are the simulated hysteresis minor loops for the field protocol for the sample structure and further modified structure, respectively. The white dots indicate the location within the hysteresis that the magnetization and power pixel plots are analyzed. (e) and (h) are the magnetization state at $-0.8 \mathrm{kOe}$ for the sample and further modified structure, respectively, where the color wheel in the top left corner shows the magnetization direction and the white arrow shows applied field direction. (f) [i-iii] and (i) [iv-vi] show the corresponding power pixel plots at -600 Oe for the sample and modified structure, respectively. A video of the simulated minor loop protocols is available in the Supplemental Material [29].

simultaneous resonance depends on the relative aspect ratios of the nanowires and is therefore tunable. It should be noted however that simply having three different sizes does not guarantee a coincidence angle and therefore the aspect ratios must be chosen carefully if this property is desirable.

\section{Minor loop states}

Thus far we have shown angular dependent FMR and extracted the switching behavior of the sublattices. By sweeping the field from a positive saturated state to negative field we found three phase transitions as each of the three sublattices 
switch at different fields. In the following the sample is saturated along the wide nanowire axis and then minor loops are performed where the range is increased after the completion of a full hysteresis loop. FMR spectra are taken on the negative to positive sweeps, for example, -200 to $200 \mathrm{Oe}$ and the next from -400 to 400 Oe. These steps are repeated increasing the maximum field value by 200 Oe each time up to 1 kOe. We aim to demonstrate reaching novel microstates using only linear magnetic field protocols. This is made possible by the different coercive fields of the nanowires allowing more selective switching than achievable in conventional kagome ASI.

Figure 5(a) is the FMR spectra of the fourth minor loop with field range -800 to 800 Oe which corresponds to the green line in the simulated hysteresis of Fig. 5(d). The white dot shows the point at -600 Oe on this minor loop. The inset and black arrow show the ASI orientation and field sweep direction. Two modes now occur: one with positive and one with negative gradient. This suggests that the microstate consists of nanowires whose magnetizations have components parallel and antiparallel with the increasing field. If the magnetization is parallel with the applied field then increasing the applied field will increase the effective field and therefore the frequency. If the magnetization is antiparallel with the field then increasing the field results in a lowering of the effective field and frequency. Figure 5(b) is the simulation for the same experiment and the labeled modes correspond to the magnetization profile in Fig. 5(e) and power pixel plots in Figs. 5(f)[i-iii]. Figure 5(e) shows the wide nanowires (dark blue) are antiparallel with the field and therefore mode [i] illustrated by the power pixel plot in Fig. 5(f) at $11.96 \mathrm{GHz}$ has a negative gradient. Modes [ii] and [iii] occur on the narrow (green) and medium (red) nanowires in Fig. 5(e), respectively, and both have a magnetization component collinear with the increasing field and therefore positive gradients. Note that for the same reasons discussed earlier the narrow nanowire mode [ii] is not detected experimentally. The power pixel plot for these modes are shown in Fig. 5(f) at $11.11 \mathrm{GHz}$ and $10.23 \mathrm{GHz}$.

Since the scope of this paper is to explore how modifying the structure can introduce ways of accessing novel microstates with simple field protocols, a second structure is simulated. Half of the wide nanowire widths are reduced by $35 \mathrm{~nm}$. This increases the coercive field of the modified nanowires, such that, under the same field protocol half of the wide nanowires remain unswitched at the start of the fourth minor loop. This can be seen by comparing the hysteresis in Fig. 5(d) (white dot) which shows a clear net magnetization in the negative direction with Fig. 5(g) (white dot) which is almost zero because the nanowires along this axis almost cancel each other out. This state can be seen even more clearly in Fig. 5(h) where now the wide sublattice consists of elements parallel with the field (blue) and elements antiparallel with the field (yellow). This results in modes [iv] and [vi] which have the same gradient but opposite sign. Mode [i] and [iv] are equivalent. The power pixel plots for both of these modes are shown in Fig. 5(i) at 11.96 and $8.98 \mathrm{GHz}$, respectively. Mode [v] is equivalent to mode [iii] with the exception of its relatively higher power. This is due to mode [i] splitting into modes [iv] and [iv]. Mode [vi] should in principle be detectable which means that FMR aided with micromagnetic simulations could be used for microstate characterization.

Modifications of both the sublattices and individual elements within them allows one to access novel microstates in a more controlled way than is possible with conventional ASI systems. By comparing the hysteresis in Figs. 5(d) and $5(\mathrm{~g})$ one can clearly see how modification allows access to different states under the same magnetic field protocol. This opens up the possibility of experimentally investigating magnetic defects effects on spin-wave spectra in a systematic and reliable way.

\section{SUMMARY}

Experimental and computational investigations of a threefold broken-symmetry kagome artificial spin ice have been carried out. The shape anisotropy modification scheme opens up reversal pathways unavailable to conventional ASI. These new pathways are reflected in the broadband FMR measurements where minor loops access microstates with nanowire magnetizations pointing in opposite directions. A discrepancy in the expected sinusoidal dependence of relative peak height as a function of angle implied an additive effect of several modes being brought close together at $45^{\circ}$. Subsequent simulations indicate the optimum angle for this particular system is $52^{\circ}$. This angle can be tuned by modifying the sublattice dimensions; it should be noted that not all combinations of sizes will result in this behavior. Transitions between regions discussed in the angular dependence section are expected to also see mode power enhancement. The angular dependence results also provide an insight into the underlying structure by considering that the higher shape anisotropy elements exhibit higher frequency overall and show smaller change in frequency as the angle is swept. We also present suggestions for creating extra modes by modifying the size of individual nanowires within a sublattice to reach different microstates under the same field protocol. The present analysis is not limited to modification by shape anisotropy. Choosing materials with different saturation magnetization or crystalline anisotropy for different sublattices could in principle produce similar effects. One could also introduce notches in the center of the nanowires to alter the coercive field while keeping the overall size of the nanowire constant between sublattices thus modifying the switching behavior and magnetization dynamics. The coercive field is more strongly affected by the width of each nanowire so one could design systems where the volume of each element is kept constant. The geometry also allows for a different number of experimentally detectable modes in each nanowire orientation thereby introducing forbidden band selectivity and mode suppression. Note that this emergent property depends on the volume difference of the nanowires. The elegance of the scheme is that different FMR spectral response can be selected by a straightforward change of magnetic field direction. The three different sublattice orientations produced highest mode frequencies at $1.5 \mathrm{kOe}$ of $17.24 \pm 0.10 \mathrm{GHz}, 16.45 \pm 0.25 \mathrm{GHz}$, and $15.40 \pm$ $0.10 \mathrm{GHz}$ having a range of $1.84 \pm 0.10 \mathrm{GHz}$ demonstrating mode tunability. For real device applications the frequency response at remnant fields may be more appropriate to consider 
where the range is $990 \pm 100 \mathrm{MHz}$. Modification of single elements provides control of the number of defects introduced into the system in a robust way compared to homogeneous ASI adding further degrees of freedom to tuning magnetization dynamics. Shape anisotropy modification may also provide more simple and robust ways of generating monopole defects compared to previous methods [37]. Magnetic defects have been shown to significantly alter the FMR [38] so the dynamics of these defects may be the focus of future work. Here we have demonstrated that purposefully breaking the rotational symmetry opens up a multitude of degrees of freedom to be exploited in the design of future magnonic based computing.

\section{ACKNOWLEDGMENTS}

T.D. was supported by the EPSRC Centre for Doctoral Training in Advanced Characterisation of Materials (Grant No. EP/L015277/1). W.R.B. and J.C.G. acknowledge funding from the Leverhulme grant (Grant No. RPG-2017-257). T.K. acknowledges that this work is supported by JSPS Strategic Young Researcher Overseas Visits Program for Accelerating Brain Circulation. L.F.C. acknowledges funding from the Leverhulme grant (No. RPG-2016-306). The simulation work and analysis was supported via Imperial College Research Computing Service [39].
[1] C. Nisoli, J. Li, X. Ke, D. Garand, P. Schiffer, and V. H. Crespi, Effective Temperature in an Interacting Vertex System: Theory and Experiment on Artificial Spin Ice, Phys. Rev. Lett. 105, 047205 (2010).

[2] E. Mengotti, L. J. Heyderman, A. F. Rodríguez, F. Nolting, R. V. Hügli, and H.-B. Braun, Real-space observation of emergent magnetic monopoles and associated Dirac strings in artificial kagome spin ice, Nat. Phys. 7, 68 (2011).

[3] S. T. Bramwell, S. R. Giblin, S. Calder, R. Aldus, D. Prabhakaran, and T. Fennell, Measurement of the charge and current of magnetic monopoles in spin ice, Nature (London) 461, 956 (2009).

[4] L. D. C. Jaubert and P. C. W. Holdsworth, Signature of magnetic monopole and Dirac string dynamics in spin ice, Nat. Phys. 5, 258 (2009).

[5] D. J. P. Morris, D. A. Tennant, S. A. Grigera, B. Klemke, C. Castelnovo, R. Moessner, C. Czternasty, M. Meissner, K. C. Rule, J.-U. Hoffmann, K. Kiefer, S. Gerischer, D. Slobinsky, and R. S. Perry, Dirac strings and magnetic monopoles in the spin ice $\mathrm{Dy}_{2} \mathrm{Ti}_{2} \mathrm{O}_{7}$, Science 326, 411 (2009).

[6] G.-W. Chern, C. Reichhardt, and C. Nisoli, Realizing threedimensional artificial spin ice by stacking planar nano-arrays, Appl. Phys. Lett. 104, 013101 (2014).

[7] Y. Perrin, B. Canals, and N. Rougemaille, Extensive degeneracy, Coulomb phase and magnetic monopoles in artificial square ice, Nature 540, 410 (2016).

[8] Z. Budrikis, P. Politi, and R. L. Stamps, A network model for field and quenched disorder effects in artificial spin ice, New J. Phys. 14, 045008 (2012).

[9] L. J. Heyderman and R. L. Stamps, Artificial ferroic systems: novel functionality from structure, interactions and dynamics, J. Phys. Condens. Matter 25, 363201 (2013).

[10] C. Nisoli, R. Wang, J. Li, W. F. McConville, P. E. Lammert, P. Schiffer, and V. H. Crespi, Ground State Lost but Degeneracy Found: The Effective Thermodynamics of Artificial Spin Ice, Phys. Rev. Lett. 98, 217203 (2007).

[11] W. R. Branford, S. Ladak, D. E. Read, K. Zeissler, and L. F. Cohen, Emerging chirality in artificial spin ice, Science 335, 1597 (2012).

[12] S. Ladak, D. Read, T. Tyliszczak, W. R. Branford, and L. F. Cohen, Monopole defects and magnetic coulomb blockade, New J. Phys. 13, 023023 (2011).
[13] V. S. Bhat, F. Heimbach, I. Stasinopoulos, and D. Grundler, Magnetization dynamics of topological defects and the spin solid in a kagome artificial spin ice, Phys. Rev. B 93, 140401(R) (2016).

[14] X. Zhou, G.-L. Chua, N. Singh, and A. O. Adeyeye, Large area artificial spin ice and anti-spin ice $\mathrm{Ni}_{80} \mathrm{Fe}_{20}$ structures: Static and dynamic behavior, Adv. Funct. Mater. 26, 1437 (2016).

[15] J. Dubowik, P. Kuświk, M. Matczak, W. Bednarski, F. Stobiecki, P. Aleshkevych, H. Szymczak, M. Kisielewski, and J. Kisielewski, Ferromagnetic resonance and resonance modes in kagome lattices: From an open to a closed kagome structure, Phys. Rev. B 93, 224423 (2016).

[16] M. B. Jungfleisch, W. Zhang, E. Iacocca, J. Sklenar, J. Ding, W. Jiang, S. Zhang, J. E. Pearson, V. Novosad, J. B. Ketterson, O. Heinonen, and A. Hoffmann, Dynamic response of an artificial square spin ice, Phys. Rev. B 93, 100401(R) (2016).

[17] E. Iacocca, S. Gliga, R. L. Stamps, and O. Heinonen, Reconfigurable wave band structure of an artificial square ice, Phys. Rev. B 93, 134420 (2016).

[18] M. Krawczyk and D. Grundler, Review and prospects of magnonic crystals and devices with reprogrammable band structure, J. Phys. Condens. Matter 26, 123202 (2014).

[19] J. Topp, D. Heitmann, M. P. Kostylev, and D. Grundler, Making a Reconfigurable Artificial Crystal by Ordering Bistable Magnetic Nanowires, Phys. Rev. Lett. 104, 207205 (2010).

[20] D. M. Arroo, J. C. Gartside, and W. R. Branford, Sculpting the Spin-Wave Response of Artificial Spin Ice via Microstate Selection, arXiv:1805.01397 [Phys. Rev. B (to be published)].

[21] R. F. Wang, C. Nisoli, R. S. Freitas, J. Li, W. McConville, B. J. Cooley, M. S. Lund, N. Samarth, C. Leighton, V. H. Crespi, and P. Schiffer, Artificial 'spin ice' in a geometrically frustrated lattice of nanoscale ferromagnetic islands, Nature (London) 439, 303 (2006).

[22] R. F. Wang, J. Li, W. Mcconville, C. Nisoli, X. Ke, J. W. Freeland, V. Rose, M. Grimsditch, P. Lammert, V. H. Crespi, and P. Schiffer, Demagnetization protocols for frustrated interacting nanomagnet arrays, J. Appl. Phys 101, 09J104 (2007).

[23] Y.-L. Wang, Z.-L. Xiao, A. Snezhko, J. Xu, L. E. Ocola, R. Divan, J. E. Pearson, G. W. Crabtree, and W.-K. Kwok, Rewritable artificial magnetic charge ice, Science 352, 962 (2015).

[24] J. C. Gartside, D. M. Burn, L. F. Cohen, and W. R. Branford, A novel method for the injection and manipulation of mag- 
netic charge states in nanostructures, Sci. Rep. 6, 32864 (2016).

[25] J. C. Gartside, D. M. Arroo, D. M. Burn, V. L. Bemmer, A. Moskalenko, L. F. Cohen, and W. R. Branford, Realization of ground state in artificial kagome spin ice via topological defectdriven magnetic writing, Nat. Nanotechnol. 13, 53 (2018).

[26] W. Bang, M. B. Jungfleisch, F. Montoncello, B. W. Farmer, P. N. Lapa, A. Hoffmann, L. Giovannini, L. E. De Long, and J. B. Ketterson, Coupled macrospins: Mode dynamics in symmetric and asymmetric vertices, AIP Adv. 8, 056020 (2018).

[27] F. Montoncello, L. Giovannini, W. Bang, J. B. Ketterson, M. B. Jungfleisch, A. Hoffmann, B. W. Farmer, and L. E. De Long, Mutual influence between macrospin reversal order and spinwave dynamics in isolated artificial spin-ice vertices, Phys. Rev. B 97, 014421 (2018).

[28] A. Vansteenkiste, J. Leliaert, M. Dvornik, M. Helsen, F. GarciaSanchez, and B. Van Waeyenberge, The design and verification of MuMax3, AIP Adv. 4, 107133 (2014).

[29] See Supplemental Material at http://link.aps.org/supplemental/ 10.1103/PhysRevB.100.054433 for sample fabrication and characterization, experimental and simulation details, as well as accompanying videos.

[30] M. Dvornik, Y. Au, and V. V. Kruglyak, Micromagnetic Simulations in Magnonics (Springer, Berlin, Heidelberg, 2013), pp. 101-115.

[31] J. Jorzick, S. O. Demokritov, B. Hillebrands, M. Bailleul, C. Fermon, K. Y. Guslienko, A. N. Slavin, D. V. Berkov, and N. L. Gorn, Spin Wave Wells in Nonellipsoidal Micrometer Size Magnetic Elements, Phys. Rev. Lett. 88, 047204 (2002).
[32] R. P. Cowburn, Property variation with shape in magnetic nanoelements, J. Phys. D 33, R1 (2000).

[33] R. Zivieri, P. Malagò, L. Giovannini, S. Tacchi, G. Gubbiotti, and A. Olusola Adeyeye, Soft magnonic modes in twodimensional permalloy antidot lattices, J. Phys.: Condens. Matter 25, 336002 (2013).

[34] G. Gubbiotti, G. Carlotti, T. Okuno, M. Grimsditch, L. Giovannini, F. Montoncello, and F. Nizzoli, Spin dynamics in thin nanometric elliptical Permalloy dots: A Brillouin light scattering investigation as a function of dot eccentricity, Phys. Rev. B 72, 184419 (2005).

[35] V. Kapaklis, U. B. Arnalds, A. Farhan, R. V. Chopdekar, A. Balan, A. Scholl, L. J. Heyderman, and B. Hjörvarsson, Thermal fluctuations in artificial spin ice, Nat. Nanotechnol. 9, 514 (2014).

[36] V. Kapaklis, U. B. Arnalds, A. Harman-Clarke, E. T. Papaioannou, M. Karimipour, P. Korelis, A. Taroni, P. C. W. Holdsworth, S. T. Bramwell, and B. Hjörvarsson, Melting artificial spin ice, New J. Phys. 14, 035009 (2012).

[37] B. K. Kuanr, R. Lopusnik, L. M. Malkinski, M. Wenger, M. Yu, D. Scherer, R. E. Camley, and Z. Celinski, High-frequency characterization of Permalloy nanosized strips using network analyzer ferromagnetic resonance, J. Appl. Phys. 103, 07 C508 (2008).

[38] S. Gliga, A. Kákay, R. Hertel, and O. G. Heinonen, Spectral Analysis of Topological Defects in an Artificial Spin-Ice Lattice, Phys. Rev. Lett. 110, 117205 (2013).

[39] https://www.imperial.ac.uk/admin-services/ict/selfservice/research-support/rcs/impact/citing-the-service/. 